\title{
Continuity conditions defined by indices of non-compactness and applications to differentiability theory
}

\author{
WARREN B. MOORS
}

Classically, the differentiability theory for continuous convex functions on Banach spaces has centred on finding and characterising those Banach spaces in which every continuous convex function is differentiable on a dense subset of its domain. However, in this thesis, we investigate the differentiability theory of continuous convex functions of Banach spaces on a function by function basis.

The key to this approach is the consideration of several new continuity conditions defined in terms of indices of non-compactness, all of which have implications for differentiability theory. Nevertheless, even with this function by function approach we are still able to give new characterisations of classes of Banach spaces in which every continuous convex function is Fréchet differentiable on a dense subset of its domain.

Chapter 1 is concerned with preliminaries; it contains the definitions and elementary properties of four indices of non-compactness. The first one is due to Kuratowski, and the second due to de Blasi, the other two are logical extension of these two indices.

In Chapter 2 we introduce several continuity conditions related to the indices and explore some of their permanence properties. But more importantly, for a significant class of set-valued mappings (minimal weak* cuscos), we are able to determine when they are single-valued norm upper semi-continuous on a dense $G_{\delta}$ subset of their domain.

The application to differentiability theory is presented in Chapter 3. We characterise the Fréchet differentiability of continuous convex functions on a Banach space in terms of the continuity conditions developed in Chapter 2. We also show that a Banach space where every continuous convex function of an open convex subset is Fréchet differentiable on a dense $G_{\delta}$ subset of its domain is characterised by every non-empty bounded set in its dual possessing slices which can be approximated arbitrarily closely by weakly compact sets.

Received 17th September, 1992.

Thesis submitted to the University of Newcastle, March 1992. Degree approved, August 1992. Supervisor: Assoicate Professor John R. Giles.

Copyright Clearance Centre, Inc. Serial-fee code: 0004-9729/93 \$A2.00+0.00. 
Next, we consider a continuity condition called GGS upper semi-continuity, which is a weaker notion than weak upper semi-continuity, and we show that it has significance in determining differentiability properties.

We also consider Banach spaces in which every point of the unit sphere is a denting point in a sense defined by our indices of non-compactness, and we show that on a space of this type, the members of a significant class of continuous convex functions on its dual have desirable differentiability properties.

In Chapter 4, we consider two geometric conditions, the Drop Property and Nearly Uniform Convexity, and we show that both of these properties can be characterised by a property of the norm which is defined in terms of a continuity condition introduced in Chapter 2. Using these characterisations we are then able to obtain new facts about spaces which possess these geometrical properties. We also consider weaker forms of these geometrical properties, namely, the weak* Drop Property and weak* Nearly Uniform Convexity and we establish a natural duality between them.

The University of Auckland

Private Bag 92019

Auckland

New Zealand 